\title{
Respiratory Syncytial Virus Infection Results in Airway Hyperresponsiveness and Enhanced Airway Sensitization to Allergen
}

\author{
Jürgen Schwarze, Eckard Hamelmann, Katherine L. Bradley, Katsuyuki Takeda, and Erwin W. Gelfand \\ Division of Basic Sciences, Department of Pediatrics, National Jewish Medical and Research Center, Denver, Colorado 80206
}

\begin{abstract}
Viral respiratory infections can predispose to the development of asthma by mechanisms that are presently undetermined. Using a murine model of respiratory syncytial virus (RSV) infection, acute infection is associated with airway hyperresponsiveness as well as enhanced responses to subsequent sensitization to allergen. We demonstrate that acute viral infection results in increased airway responsiveness to inhaled methacholine and pulmonary neutrophilic and eosinophilic inflammation. This response is associated with predominant production of Th-1-type cytokines in peribronchial lymph node cells in vitro. Mice sensitized to ovalbumin via the airways after RSV infection developed increased airway responsiveness to methacholine and pulmonary eosinophilic and neutrophilic inflammation, associated with the predominant production of Th-2-type cytokines. Treatment of the mice with anti-IL-5 antibody abolished airway hyperresponsiveness and eosinophilic but not neutrophilic inflammation in both acutely infected mice and mice sensitized after infection. We conclude that RSV infection results in airway hyperresponsiveness in the acute phase and leads to changes in immune function that can enhance the effects of airway sensitization to antigen after infection. In both situations, airway hyperresponsiveness is closely associated with pulmonary eosinophilic inflammation. This model provides a means for further analyzing the influence of viral respiratory infections on airway sensitization and the development of altered airway responsiveness. (J. Clin. Invest. 1997. 99:226-233.) Key words: respiratory syncytial virus • mice, inbred BALB/c • respiratory hypersensitivity • airway obstruction $\cdot$ pulmonary eosinophilia
\end{abstract}

\section{Introduction}

Viral respiratory tract infections can cause increased bronchial reactivity in normal subjects and can exacerbate preexisting asthma $(1,2)$. Respiratory viruses are the most important trigger for acute asthma symptoms (3). Among the respiratory viruses implicated in childhood asthma are respiratory syncytial

Address correspondence to Erwin W. Gelfand, M.D., Department of Pediatrics, National Jewish Medical and Research Center, 1400 Jackson Street, Denver, Colorado 80206. Phone: 303-398-1196; FAX: 303270-2105; E-mail: gelfande@njc.org

Received for publication 23 October 1996 and accepted in revised form 9 April 1997.

J. Clin. Invest.

(C) The American Society for Clinical Investigation, Inc.

0021-9738/97/07/0226/08 \$2.00

Volume 100, Number 1, July 1997, 226-233 virus (RSV), ${ }^{1}$ the most common respiratory virus in the age group of $<2 \mathrm{yr}$, rhinoviruses, parainfluenza virus type III, and coronavirus (4). Epidemiological evidence suggests that viral respiratory infections may contribute to allergic sensitization (5). A prospective cohort study with matched controls showed that RSV bronchiolitis in infancy was the most important risk factor for the development of asthma together with allergen specific serum $\operatorname{IgE}$ by the age of $3 \mathrm{yr}$ (6). This evidence raises the possibility that there may be synergistic immunological interactions after viral respiratory infections and allergic airway sensitization. The mechanisms underlying such interactions have not been well-defined, although many potential mechanisms have been suggested for the exacerbation of asthma by viral infection $(3,7)$.

To investigate the cellular mechanisms linking viral respiratory infections and allergic airway sensitization, animal models are required as such studies cannot be performed easily in humans. Guinea pigs and other animals (cotton rats and lambs) have been used to study airway responsiveness (AR) and pulmonary changes during respiratory virus infection (811). The immunology of RSV infections has been studied extensively in the mouse (12-14) and murine models to investigate the effect of RSV infection on allergic airway sensitization have shown that sensitization to antigen by nasal instillation or by aerosol inhalation during acute RSV infection is associated with an increase in the production of allergen-specific immunoglobulins, especially $\operatorname{IgE}(15,16)$. A murine model that additionally allows the investigation of $\mathrm{AR}$ and assessment of pulmonary inflammation in this setting has not been reported. Here, we describe the consequences of acute RSV infection and allergic sensitization on the airway inflammatory response and on AR to inhaled methacholine (MCh) measured by barometric whole body plethysmography (17).

\section{Methods}

Animals. Female BALB/c mice, $8-12$ wk of age, free of specific pathogens, were obtained from Jackson Laboratories (Bar Harbor, $\mathrm{ME}$ ). The mice were maintained on ovalbumin (OVA) free diets. All experimental animals used in this study were under a protocol approved by the Institutional Animal Care and Use Committee of the National Jewish Center for Immunology and Respiratory Medicine.

Virus. Human respiratory syncytial virus A (long strain), free of chlamydia contamination, was obtained from the Viral Diagnostics Laboratory, Health Sciences Center, University of Colorado (Denver, $\mathrm{CO}$ ). The virus was cultured on Hep 2 cells from American Type Culture Collection (ATCC) (Rockville, MD) in medium containing FCS from GIBCO BRL (Gaithersburg, MD). It was purified as described (18). Briefly, cells and supernatant were harvested, the cells were disrupted by ultrasonic manipulation, and the suspension was

1. Abbreviations used in this paper: $\mathrm{AR}$, airway responsiveness; $\mathrm{EU}$, ELISA units; MCh, methacholine; OVA, ovalbumin; PBLN, peribronchial lymph nodes; Penh, enhanced pause; PFU, plaque-forming units; RSV, respiratory syncytial virus. 
clarified by centrifugation $(2,500 \mathrm{rpm}, 20 \mathrm{~min})$. The supernatant was layered over $30 \%$ sucrose in STEU buffer (sodium chloride $0.1 \mathrm{M}$, Tris $0.01 \mathrm{M}$, EDTA $0.001 \mathrm{M}$, and urea $1 \mathrm{M}$; all obtained from Sigma Chemical Co. [St. Louis, MO]) and centrifuged $\left(100,000 \mathrm{~g}, 1 \mathrm{~h}, 10^{\circ} \mathrm{C}\right)$. The pellet was resuspended in $1.2 \mathrm{ml}$ PBS, aliquoted, and frozen at $-70^{\circ} \mathrm{C}$. The suspension contained $4 \times 10^{7}$ plaque-forming units (PFU) of RSV per milliliter as assessed by quantitative plaque-forming assay.

To inactivate the virus, an aliquot of the suspension was irradiated with ultraviolet light for $15 \mathrm{~min}$ on ice (19). A protein assay was performed before and after the UV inactivation to adjust for any dilutional changes due to minimal evaporation. After irradiation, no evidence of viable RSV could be detected by plaque-forming assay.

Infection of mice. Mice were infected under light anesthesia (Avertin $2.5 \%, 0.015 \mathrm{ml} / \mathrm{g}$ body weight) by intranasal inoculation of RSV $\left(10^{5}\right.$ PFU in $50 \mu l$ PBS). Controls were sham infected with PBS or UV-inactivated RSV (equivalent of $10^{5} \mathrm{PFU}$ ) in the same way. Efficacy of this infection procedure was regularly tested by qualitative plaque-forming assays (20). Briefly, on day 4 postinfection mice were killed, the lungs were removed, homogenized, and centrifuged, and the supernatant was added to Hep 2 cell cultures. Infection could be demonstrated in all infected animals tested but not in mice sham infected with either PBS or UV-inactivated RSV.

Experimental protocols. Mice were infected on day 0. AR was assessed on day 6,12 , or 21 postinfection and animals were killed the next day for collection of blood and the removal of peribronchial lymph nodes (PBLN) and lungs. In sensitization experiments, mice were sensitized by inhalation of ovalbumin (OVA) obtained from Sigma Chemical Co. (1\% in $7 \mathrm{ml}$ PBS, $20 \mathrm{~min} / \mathrm{d}$ ) for 10 consecutive days starting on day 11 or day 21 postinfection using an AeroSonic Nebulizer 5000 D from DeVilbiss (Sommerset, PA), as described (21). An abbreviated sensitization protocol (5 d, starting on day 16 postinfection) was also tested. AR was assessed on day 21 or day 31 postinfection and mice were killed the next day.

In a separate set of experiments TRFK-5, a DNAX, antibody directed against IL-5, (kindly provided by Dr. R. Coffman, Palo Alto, $\mathrm{CA}$ ) or rat $\mathrm{IgG}$ as a control (Sigma Chemical Co.) were administered intravenously ( $150 \mu \mathrm{g}$ per dose) on days $-1,1$, and 4 of acute infection, or on days 14,16 , and 18 postinfection during the 10-d sensitization period.

\section{Determination of $A R$}

AR was assessed by a method we have described recently (17), using a single chamber whole body plethysmograph obtained from Buxco (Troy, NY). In this system, an unrestrained, spontaneously breathing mouse is placed into the main chamber of the plethysmograph and pressure differences between this chamber and a reference chamber are recorded. The resulting box pressure signal is caused by volume and resultant pressure changes during the respiratory cycle of the animal. A low-pass filter in the wall of the main chamber allows thermal compensation. From these box pressure signals the phases of the respiratory cycle, tidal volumes, and the enhanced pause (Penh) can be calculated. Penh is a dimensionless value that represents a function of the proportion of maximal expiratory to maximal inspiratory box pressure signals and of the timing of expiration. It correlates closely with pulmonary resistance measured by conventional two-chamber plethysmography in ventilated animals (17). Penh was used as the measure of airway responsiveness in this study. In the plethysmograph, mice were exposed for $3 \mathrm{~min}$ to nebulized PBS and subsequently to increasing concentrations of nebulized methacholine (Sigma Chemical Co.) in PBS using the AeroSonic ultrasonic nebulizer. After each nebulization, recordings were taken for $3 \mathrm{~min}$. The Penh values measured during each 3 min sequence were averaged and are expressed for each methacholine concentration as the percentage of baseline Penh values after PBS exposure.

Lung cell isolation. Lung cells were isolated by collagenase digestion as described previously (22) and counted with a hemocytometer. Cytospin slides were stained with Leukostat from Fisher Diag- nostics (Pittsburgh, PA) and differential cell counts were performed in a blinded fashion by counting at least 300 cells under light microscopy.

Cell preparation. PBLN were harvested and mononuclear cells were purified by passing the tissue through a stainless steel mesh, followed by density gradient centrifugation (Organon Teknika-Cappel, Durham, NC). Cells were washed three times with PBS and resuspended in RPMI 1640 medium (GIBCO BRL).

In-vitro cytokine production. Mononuclear cells were cultured for $48 \mathrm{~h}$ in 96-well round-bottom plates at a concentration of 400,000 cells per well in the presence or absence of OVA $(10 \mu \mathrm{g} / \mathrm{ml})$, UVinactivated RSV (equivalent of 20,000 PFU per well) or the combination of ionomycin $\left(\mathrm{I}^{1}, 0.5 \mu \mathrm{M}\right)$ from Calbiochem Corp. (La Jolla, CA) and phorbol 12,13-dibutyrate ( $\mathrm{P}^{1}, 10 \mathrm{ng} / \mathrm{ml}$; Sigma Chemical Co.). Supernates were harvested and frozen at $-20^{\circ} \mathrm{C}$. The concentrations of IFN- $\gamma$, IL-4, and IL-5 in the supernates were assessed by ELISA as described (23). Briefly, Immulon-2 plates from Dynatech Laboratories, Inc. (Chantilly, VA) were coated with anti-IFN- $\gamma$ (R4-6A2; PharMingen, San Diego, CA), anti-IL-4 (11B11; PharMingen) or anti-IL-5 antibodies (TRFK-5; gift of Dr. R. Coffman) and blocked with $\mathrm{PBS} / 10 \%$ FCS overnight. Samples were added; biotinylated anti-IFN- $\gamma$ (XMG 1.2; PharMingen), anti-IL-4 (BVD6-24G2; PharMingen), or anti-IL-5 antibodies (TRFK-4; PharMingen) were used as detecting antibodies and the reactions were amplified with avidinhorseradish-peroxidase (Sigma Chemical Co.). Cytokine levels were calculated by comparison with known cytokine standards (PharMingen). The limit of detection in the assay was $4 \mathrm{pg} / \mathrm{ml}$ for each cytokine.

Measurement of anti-OVA antibody and total Ig levels. Total IgG and IgE levels and anti-OVA IgE and IgG1 antibody levels in serum were measured by ELISA as described previously (21). The antiOVA antibody titers of samples were related to internal pooled standards and expressed as ELISA units (EU). Total IgG and IgE levels were calculated by comparison with known mouse $\mathrm{IgG}$ or $\mathrm{IgE}$ standards (PharMingen). The limit of detection was $100 \mathrm{pg} / \mathrm{ml}$ for $\mathrm{IgE}$ and $1 \mathrm{ng} / \mathrm{ml}$ for $\mathrm{IgG}$.

Statistical analysis. Pairs of groups were compared by Student's $t$ test, comparison of more than two groups was performed by the Tukey-Kramer HSD test, and Pearson correlations were performed. $P$ values for significance were set at 0.05 . Values for all measurements are expressed as the mean \pm SD except for values of AR (Penh) which are expressed as the mean \pm SEM.

\section{Results}

Acute RSV infection causes increased airway reactivity to methacholine. BALB/c mice were infected by intranasal instillation of RSV $\left(10^{5} \mathrm{PFU}\right)$. The airway response to $\mathrm{MCh}$ challenge in mice infected with RSV and in sham-infected controls was assessed by barometric whole-body plethysmography on day 6 of the acute infection. The airways of mice infected with RSV were significantly more reactive than the airways of shaminfected controls (Fig. $1 \mathrm{~A}$ ). The Penh to $50 \mathrm{mg} / \mathrm{ml} \mathrm{MCh}$ increased 5.33 \pm 2.41 -fold over PBS in infected mice compared to a $2.75 \pm 1.42$-fold increase in mice sham infected with PBS. This increase in AR in infected mice could still be demonstrated $12 \mathrm{~d}$ after infection, but returned to normal levels by day 21 postinfection (Fig. $1 \mathrm{~B}$ ). Mice exposed to UV-inactivated RSV did not show any increase in AR (Fig. $1 A$ ).

Acute RSV infection triggers influx of pulmonary eosinophils and neutrophils. To investigate changes in pulmonary inflammatory cells during the acute RSV infection, lung cells were isolated and differential counts performed. In mice acutely infected with RSV (day 6), the numbers of eosinophils and neutrophils were increased significantly in lung cell isolates compared to sham infected controls (Fig. 2). The in- 

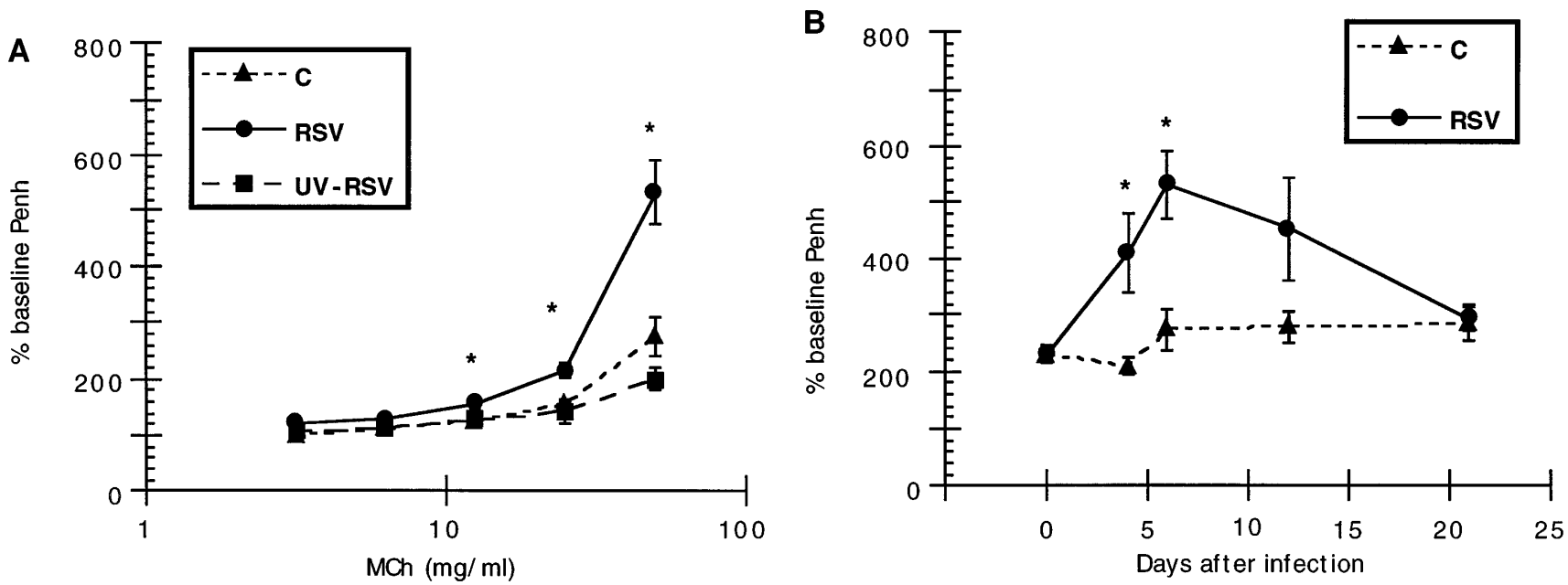

Figure 1. (A) Acute RSV infection results in increased airway responsiveness to MCh. Mice were infected with RSV $(R S V, n=16)$, sham infected with PBS $(C, n=16)$, or with UV-inactivated RSV $(U V-R S V, n=6)$. On day 6 postinfection AR to increasing concentrations of inhaled $\mathrm{MCh}(3-50 \mathrm{mg} / \mathrm{ml})$ was assessed by barometric body plethysmography and Penh values were calculated. Means \pm SEM of Penh values from four independent experiments are expressed as the percentage of baseline Penh values observed after PBS exposure. *Significant differences, $P<$ 0.05. (B) Time course of airway responsiveness after acute RSV infection. Airway responsiveness was assessed on days 4, 12, and 21 postinfection. Illustrated are mean \pm SEM of Penh values at $50 \mathrm{mg} / \mathrm{ml} \mathrm{MCh}$ on these days comparing mice infected with RSV $(R S V, n=12)$ to mice sham infected with PBS $(C, n=12)$ from three independent experiments per time point. *Significant differences, $P<0.05$.

creases in numbers of eosinophils and neutrophils were 1.87and 2.57-fold, respectively. The total numbers of isolated lung cells did not differ significantly between the groups. $21 \mathrm{~d}$ after infection, the numbers of eosinophils and neutrophils in the lung returned to control levels.

Acute RSV infection causes a transient increase in IFN- $\gamma$ production and a decrease in IL-4 and IL-5 production. We measured the production of IFN- $\gamma$, IL-4, and IL-5 in 48-h cultures of mononuclear cells obtained from PBLN, harvested on day 6 postinfection and stimulated with phorbol 12,13-dibutyrate/ionomycin (P/I). Production of IFN- $\gamma$ was increased significantly whereas levels of IL-4 and IL-5 were decreased significantly (Fig. 3). Parallel findings were observed in cultures stimulated with RSV. By day 12 and 21 postinfection, cytokine levels returned to normal (data not shown). These findings

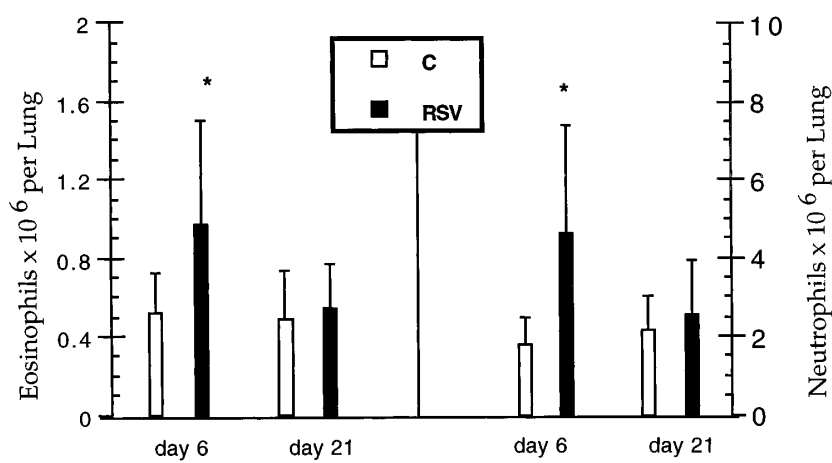

Figure 2. Acute RSV infection results in eosinophil and neutrophil influx into the lung. Lung cells were isolated from mice infected with $\operatorname{RSV}(n=12)$ or sham infected with PBS $(C, n=12)$ on days 6 and 21 postinfection. Numbers of eosinophils and neutrophils per lung were determined. Illustrated are means \pm SD of the numbers of these cells from four independent experiments. *Significant differences, $P<0.05$. suggest that acute RSV infection leads to an increased production of Th-1-type cytokines and a decrease in the production of Th-2-type cytokines by mononuclear cells from PBLN. Moreover, these changes are transient.

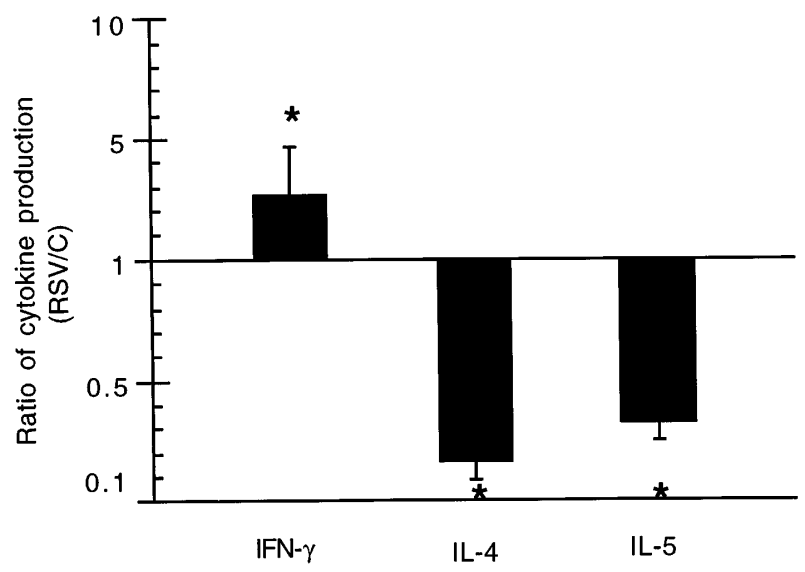

Figure 3. Acute RSV infection results in a predominant Th-1-type cytokine response in PBLN. PBLN were harvested on day 6 postinfection from mice infected with RSV $(n=12)$ or sham infected with PBS $(n=12)$. Mononuclear cells were isolated and cultured in the presence of P/I for $48 \mathrm{~h}$. Concentrations of IFN- $\gamma$, IL-4, and IL-5 were assessed in culture supernatants by ELISA. The means \pm SD of the ratios of cytokine concentrations comparing RSV exposed mice to PBS exposed mice from three independent experiments are illustrated. *Significant differences between the groups in the absolute concentrations of IFN- $\gamma$ (PBS: $4269.6 \pm 2654.8 \mathrm{pg} / \mathrm{ml}$; RSV: $7860.8 \pm 1901.7$ $\mathrm{pg} / \mathrm{ml}, * P=0.04)$, IL-4 (PBS: $71.0 \pm 24.7 \mathrm{pg} / \mathrm{ml}$; RSV: $10.7 \pm 1.55$ $\mathrm{pg} / \mathrm{ml}, * P=0.02$ ), and IL-5 (PBS: $1996.3 \pm 536.7 \mathrm{pg} / \mathrm{ml}$; RSV: $686.0 \pm 322.8 \mathrm{pg} / \mathrm{ml} ; * P=0.03)$. 
A
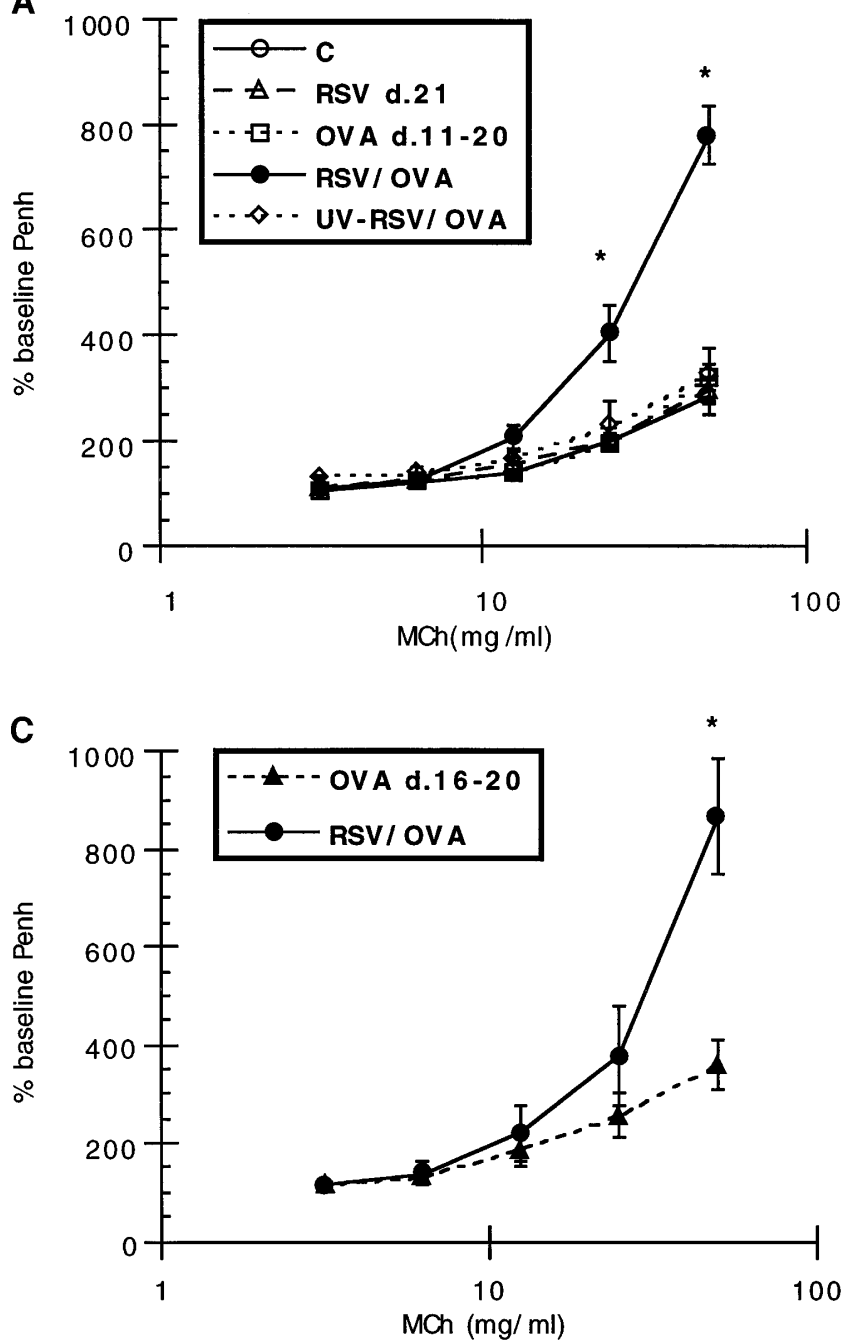

RSV infection before OVA sensitization results in increased airway responsiveness to methacholine. To investigate the effects of RSV infection on subsequent sensitization of the airways to inhaled OVA, two experimental approaches were used: mice were infected with RSV on day 0 and either exposed daily to nebulized OVA from day 11 to day 20 postinfection when effects of the acute infection could still be observed, or OVA exposure was begun only after complete resolution of the effects of acute RSV infection (days 21-30 postinfection). The airway response to $\mathrm{MCh}$ was assessed by whole body plethysmography on day 21 or day 31 postinfection. Mice infected with RSV before exposure to OVA aerosol for $10 \mathrm{~d}$ (days 11-20 or 21-30 postinfection) showed significant increases in AR (Fig. 4, $A$ and $B$ ). In these mice, Penh was significantly increased over controls. Sensitization to OVA via the airways over $10 \mathrm{~d}$ by itself failed to significantly increase responsiveness to $\mathrm{MCh}$ when monitored by whole body plethysmography. This is in contrast to the development of airway hyperresponsivness as assessed by the response of tracheal smooth muscle segments to electrical field stimulation using this protocol (21). Even mice exposed to OVA for as short a period as $5 \mathrm{~d}$ after RSV infection showed significant increases in Penh (Fig. 4 C). These results indicate that previous infection

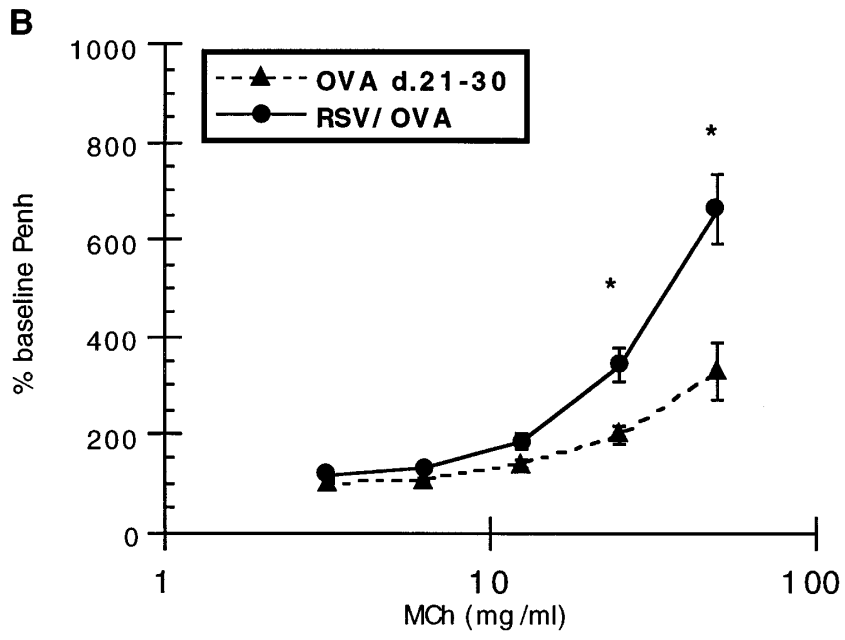

Figure 4. Airway sensitization after RSV infection results in increased airway responsiveness to MCh. Mice were infected with RSV with or without OVA sensitization ( $R S V ; R S V / O V A)$. Controls were sham infected with PBS $(C ; O V A)$ or with UV-inactivated RSV $(U V-R S V / O V A)$. $(A)$ From day 11 to day 20 postinfection, mice were exposed daily to nebulized OVA (1\%, $20 \mathrm{~min})(O V A, n=12 ; R S V / O V A, n=12$, $U V-R S V / O V A, n=8)$ or to nebulized PBS $(C, n=12 ; R S V, n=12)$ as a control. $(B)$ Delayed airway sensitization after RSV infection results in increased airway responsiveness to MCh. (C) Abbreviated airway sensitization after RSV infection results in increased airway responsiveness to $\mathrm{MCh}$. In separate experiments, mice were exposed to nebulized OVA (OVA, $n=8 ; R S V I O V A, n=8)$ from days 21 to 30 or $(C)$ from days 16 to 20 postinfection $(O V A, n=8 ; R S V / O V A, n=8)$. AR to increasing concentrations of inhaled $\mathrm{MCh}(3-50 \mathrm{mg} / \mathrm{ml})$ was assessed on day 21 ( $A$ and $C$ ) or day $31(B)$ postinfection by barometric wholebody plethysmography and Penh values were calculated. Means \pm SEM of Penh values from four $(A)$ or three $(B$ and $C)$ independent experiments are expressed as the percentage of baseline Penh values observed after PBS exposure. *Significant differences, $P<0.05$.

with RSV resulted in enhanced AR after OVA sensitization, even if the exposure to OVA was initiated after the effects of RSV had subsided. Further, infectious RSV was required as noninfectious virus did not elicit these effects (Fig. 4A).

Pulmonary eosinophil and neutrophil infiltration in the lungs correlates with increased airway responsiveness. The lungs of mice infected with RSV before OVA exposure for $10 \mathrm{~d}$ showed a significant increase in the numbers of eosinophils and neutrophils compared to the lungs of control mice or mice exposed to OVA alone (Fig. 5). The increases in eosinophils $(\sim 2.7$-fold $)$ and neutrophils $(\sim 2.5$-fold $)$ over control levels were similar and correlated with increases in AR to MCh. This correlation could also be demonstrated if the allergen exposure was begun after the resolution of the RSV infection or if it was shortened to $5 \mathrm{~d}$ (Table I). The numbers of pulmonary eosinophils and neutrophils of mice exposed to UV-inactivated RSV before OVA exposure did not differ from the control groups (data not shown).

Alterations in cytokine production after OVA sensitization after RSV infection. PBLN were removed on day 21 postinfection and the production of IFN- $\gamma$, IL-4 and IL-5 was assayed in supernatants of cells cultured in the presence or absence of OVA. Fig. 6 illustrates the relative changes in OVA- 


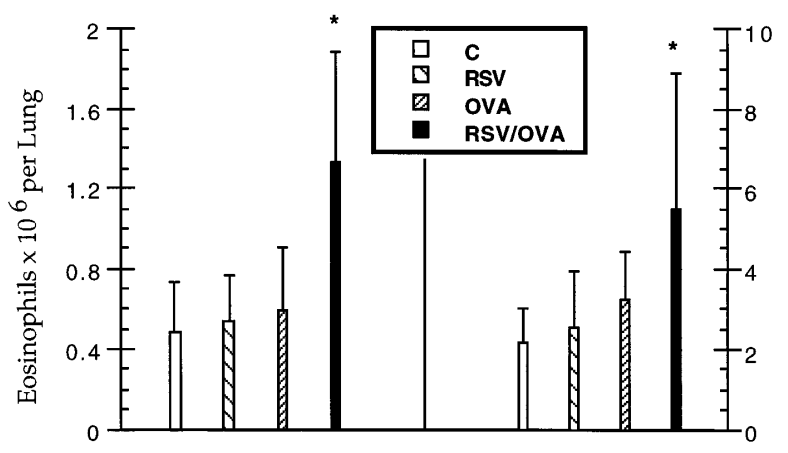

Figure 5. Airway sensitization after RSV infection results in eosinophil and neutrophil influx into the lung. Lung cells were isolated on day 21 postinfection from mice infected with RSV $(R S V, n=12)$ or sham infected with PBS $(C, n=12)$ or from mice sensitized to OVA via the airways after RSV infection ( $R S V / O V A, n=16)$ or sham infection $(O V A, n=16)$. Numbers of eosinophils and neutrophils per lung were determined. Illustrated are means \pm SD of the numbers of these cells from four independent experiments. *Significant differences, $P<0.05$.

stimulated cytokine production comparing mice that were sensitized to OVA after RSV infection to mice which were exposed to OVA after sham infection. In animals infected with RSV before exposure to OVA, the production of IFN- $\gamma$ was significantly decreased to $20.63 \pm 12.7 \mathrm{pg} / \mathrm{ml}$ from $63.3 \pm 15.49 \mathrm{pg} / \mathrm{ml}$ in mice exposed to OVA alone $(P=0.02)$. In contrast, the production of IL-4 was significantly increased from $1.08 \pm 0.13$ $\mathrm{pg} / \mathrm{ml}$ (OVA alone) to $5.33 \pm 1.08 \mathrm{pg} / \mathrm{ml}$ (RSV/OVA) $(P=$ 0.02 ). We could not detect any significant differences in the production of IL-5: $11.5 \pm 7.92 \mathrm{pg} / \mathrm{ml}$ (OVA), $8.37 \pm 4.99 \mathrm{pg} / \mathrm{ml}$ (OVA/RSV).

$R S V$ infection before airway sensitization with $O V A$ does not enhance $O V A$-specific antibody production. We monitored total IgE and IgG and OVA-specific IgE and IgG1 in the sera of mice from the different groups. The serum levels of total $\mathrm{IgE}$ and total $\mathrm{IgG}$ did not differ significantly between any of the groups. OVA-specific IgE and IgG1 could only be detected in the groups that had been exposed to OVA, but RSV

Table I. Increases in Numbers of Eosinophils in the Lung Correlate with Increases in Airway Responsiveness to MCh in Mice Sensitized to OVA After RSV Infection

\begin{tabular}{lrrc}
\hline \multicolumn{1}{c}{ Group } & \multicolumn{1}{c}{ Penh $_{50}$} & \multicolumn{1}{c}{ Eosinophils } & Correlation Eos/Penh \\
\hline OVA, days 11-20 & $3.20 \pm 0.25$ & $0.59 \pm 0.31$ & $* r=0.89$ \\
RSV/OVA & $\ddagger 7.80 \pm 0.54$ & $\ddagger 1.33 \pm 0.55$ & \\
OVA, days 21-30 & $3.29 \pm 0.57$ & $0.41 \pm 0.22$ & $* r=0.96$ \\
RSV/OVA & $\ddagger 6.62 \pm 0.73$ & $\ddagger 1.27 \pm 0.18$ & \\
OVA, days 16-20 & $3.57 \pm 0.51$ & $0.77 \pm 0.32$ & $* r=0.79$ \\
RSV/OVA & $\ddagger 8.68 \pm 3.33$ & $\ddagger 1.20 \pm 0.30$ & \\
\end{tabular}

Mice were sensitized to OVA via the airways on days 11-20, 21-30, or 16-20 after RSV infection (RSV/OVA) or sham infection with PBS $(O V A)$. AR (Penh after $50 \mathrm{mg} / \mathrm{ml} \mathrm{MCh}$, expressed as mean \pm SEM) was correlated with numbers of lung eosinophils (expressed as mean \pm SD) and the Pearson correlation coefficient $(r)$ was calculated $(* P<0.001)$. The data were obtained from three independent experiments for each sensitization protocol. ${ }^{*}$ Significant difference to the value above, $P<0.05$.

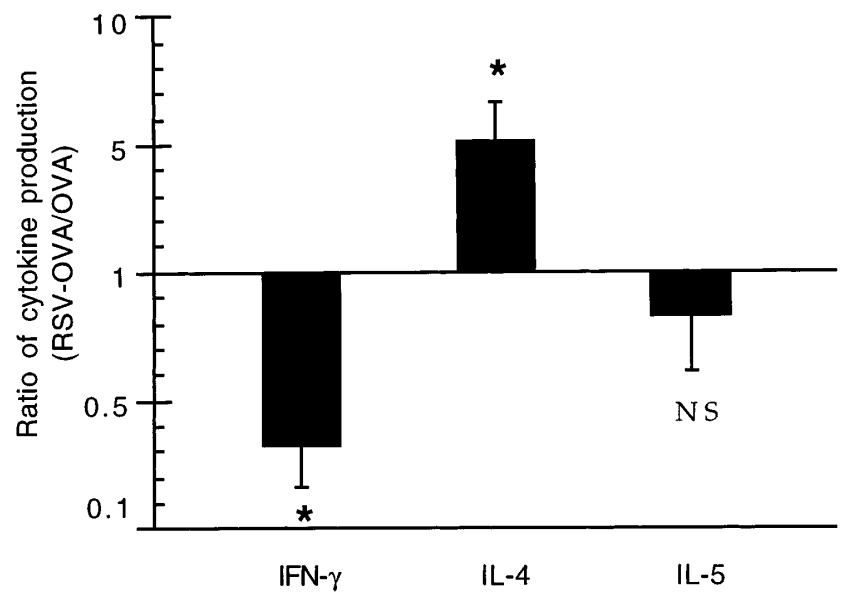

Figure 6. Airway sensitization after RSV infection results in a predominant Th-2-type cytokine response in PBLN. PBLN were harvested on day 21 postinfection from mice infected with RSV $(n=12)$ or sham infected with PBS $(n=12)$ and subsequently sensitized to nebulized OVA. Mononuclear cells were isolated and cultured in the presence of OVA $(10 \mu \mathrm{g} / \mathrm{ml})$ for $48 \mathrm{~h}$. Concentrations of IFN- $\gamma$, IL-4, and IL-5 were assessed in culture supernatants by ELISA. Shown are the means \pm SD of the ratios of cytokine concentrations comparing RSV-infected and OVA-sensitized mice to sham-infected and OVAsensitized mice from three independent experiments. *Significant differences between the groups in the absolute concentrations of IFN- $\gamma$ $(P=0.02)$ and IL-4 $(P=0.02)$.

infection before OVA inhalation did not enhance the production of OVA-specific IgE or IgG1 (Table II).

Treatment with anti-IL-5 antibody abolishes eosinophil inflammation and normalizes airway function. In both acute RSV infection and RSV infection followed by OVA sensitization, increased numbers of eosinophils and neutrophils were observed in lung digests. To define the role of eosinophils in the development of altered AR to MCh in acutely infected mice and in mice that had been infected with RSV before OVA sensitization, mice were treated with anti-IL-5 antibody (or rat IgG as a control) either during acute RSV infection on days

Table II. RSV Infection Before Sensitization with OVA Does Not Enhance Production of OVA-specific Antibodies

\begin{tabular}{lcccc}
\hline \multicolumn{1}{c}{ Group } & $\begin{array}{c}\text { Total IgE } \\
(\mathrm{ng} / \mathrm{ml})\end{array}$ & $\begin{array}{c}\text { Total IgG } \\
(\mathrm{ng} / \mathrm{ml})\end{array}$ & $\begin{array}{c}\text { OVA-specific } \\
\text { IgE (EU/ml) }\end{array}$ & $\begin{array}{c}\text { OVA-specific } \\
\text { IgG1 (EU/ml) }\end{array}$ \\
\hline $\mathrm{C}$ & $16.7 \pm 7.3$ & $163.6 \pm 38.5$ & $<10$ & $<10$ \\
RSV day 21 & $19.9 \pm 8.3$ & $159.2 \pm 40.1$ & $<10$ & $<10$ \\
OVA & $16.5 \pm 7.6$ & $156.7 \pm 27.8$ & $* 51.3 \pm 28.0$ & $* 104.7 \pm 78.1$ \\
RSV/OVA & $17.1 \pm 9.5$ & $139.7 \pm 20.4$ & $* 48.7 \pm 20.4$ & $* 81.4 \pm 39.3$
\end{tabular}

Mice were infected with RSV $(R S V)$ or sham infected $(C)$ before exposure to nebulized PBS for $10 \mathrm{~d}$, or they were sensitized to OVA via the airways after RSV infection (RSV/OVA) or sham infection $(O V A)$. Serum was obtained on day 21 postinfection in all animals and levels of total $\mathrm{IgE}$ and $\mathrm{IgG}$ and levels of $\mathrm{IgE}$ and $\mathrm{IgG} 1$ specific for OVA were assessed by ELISA. Expressed are means \pm SD from four independent experiments $(n=12$ per group). *Significant differences to C and RSV groups $(P<0.05)$. There were no significant differences between the OVA and RSV/OVA groups. 

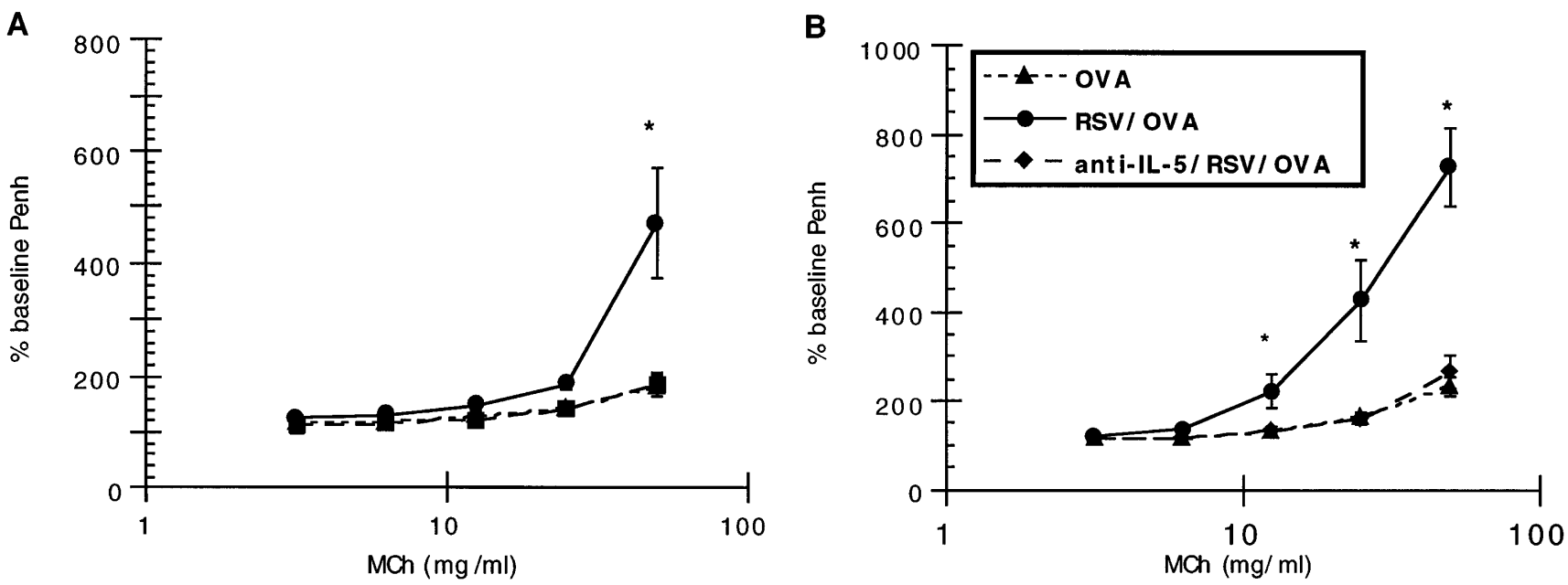

Figure 7. Anti-IL-5 antibody treatment results in normal airway responsiveness in acute RSV infection and after RSV infection and airway sensitization. (A) Mice were infected with RSV ( $R S V$, circle, $n=8$; anti-IL-5/RSV, square, $n=8)$ or sham infected with PBS $(C$, triangle, $n=8)$ on day 0 . On days $-1,1$, and 4 of infection mice were treated with anti-IL-5 antibody (TRFK-5, $150 \mu \mathrm{g}$ per dose) $($ anti-IL-5/RSV) or with rat IgG $(R S V)$ as a control. $(B)$ Anti-IL-5 antibody treatment prevents airway hyperresponsiveness after airway sensitization following RSV infection. Mice were exposed to nebulized OVA from day 11 to 20 after RSV infection $(R S V / O V A$, circle, $n=8$; anti-IL-5/RSV/OVA, diamond, $n=8)$ or after PBS $(O V A$, triangle, $n=8)$ and treated with anti-IL-5 antibody (anti-IL-5/RSV/OVA) or rat IgG $(R S V / O V A)$ as a control during the sensitization on days 14,16 , and 18 postinfection. AR to increasing concentrations of inhaled $\mathrm{MCh}(3-50 \mathrm{mg} / \mathrm{ml})$ was assessed on day $6(A)$ or on day $21(B)$ postinfection by barometric whole body plethysmography, and Penh values were calculated. Means \pm SEM of Penh values from three independent experiments are expressed as the percentage of baseline Penh values observed after PBS exposure. *Significant differences, $P<0.05$.

$-1,1,4$, or during the period of OVA sensitization after RSV infection on days 14,16 , or 18 . In both sets of experiments, administration of anti-IL-5 antibody, but not of control rat IgG, significantly reduced the numbers of eosinophils in lung cell isolates to levels below those seen in control mice or mice sensitized to OVA alone. Anti-IL-5 antibody did not alter neutro-

Table III. Anti-IL-5 Treatment Reduces AR and Lung Eosinophils but Not Neutrophils in Acute RSV Infection and in Airway Sensitization After RSV Infection

\begin{tabular}{|c|c|c|c|c|}
\hline Group & Penh $_{50}$ & Eosinophils & Neutrophils & $\begin{array}{c}\text { Correlation } \\
\text { Eos/Penh }\end{array}$ \\
\hline $\mathrm{C}$ & $1.82 \pm 0.19$ & $0.60 \pm 0.10$ & $1.60 \pm 0.59$ & \\
\hline RSV & $* 4.72 \pm 0.98$ & $* 0.94 \pm 0.48$ & $* 2.84 \pm 1.16$ & \\
\hline Anti-IL-5/RSV & $* 1.86 \pm 0.21$ & $* 0.22 \pm 0.18$ & $2.73 \pm 0.86$ & ${ }^{\ddagger} r=0.81$ \\
\hline OVA & $2.33 \pm 0.22$ & $0.59 \pm 0.16$ & $1.73 \pm 0.49$ & \\
\hline RSV/OVA & $* 7.27 \pm 0.89$ & $* 1.20 \pm 0.52$ & $* 3.33 \pm 1.58$ & \\
\hline Anti-IL-5/RSV/OVA & $* 2.63 \pm 0.37$ & $* 0.16 \pm 0.10$ & $3.27 \pm 1.18$ & ${ }^{\ddagger} r=0.81$ \\
\hline
\end{tabular}

Mice were treated with anti-IL-5 antibody either during acute RSV infection $(A n t i-I L-5 / R S V)$ or during airway sensitization to OVA after RSV infection (Anti-IL-5/RSV/OVA). They were either compared to mice which were acutely infected with RSV $(R S V)$ or sham infected with PBS $(C)$ or to mice that were sensitized to OVA via the airways after RSV infection $(R S V / O V A)$ or sham infection $(O V A)$. AR (Penh after $50 \mathrm{mg} / \mathrm{ml} \mathrm{MCh}$ ) and numbers of eosinophils and neutrophils from lung cell isolates were determined. Expressed are means \pm SD of eosinophil and neutrophil numbers per lung $\left(/ 10^{6}\right)$ and means \pm SEM of Penh values from three independent experiments each ( $n=8$ per group). *Significant difference to the value above $(P<0.05)$. Pearson correlation coefficients $(r)$ were calculated correlating Penh values with numbers of eosinophils $\left({ }^{\ddagger} P<0.001\right)$. phil numbers (Table III). In parallel, the increases in AR to MCh seen in mice infected with RSV or in mice infected and then sensitized to OVA were prevented by the anti-IL-5 treatment (Fig. 7, $A$ and $B$ ) and this correlated with eosinophil numbers (Table III). These results indicate that IL-5 is necessary for the development of pulmonary eosinophilia and that the eosinophil inflammation or IL-5 itself is critical in the development of increased $\mathrm{AR}$ in response to both acute infection with RSV and to allergen sensitization via the airways after RSV infection.

\section{Discussion}

In this study, we monitored AR after RSV infection in a wellcharacterized murine model of allergic sensitization. In this approach, RSV infection preceded airway sensitization to OVA. AR to aerosolized MCh was assessed using barometric whole body plethysmography in unrestrained animals. This model was used to assess two specific issues: $(a)$ whether acute RSV infection results in increased AR to $\mathrm{MCh}$, and $(b)$ whether RSV infection can facilitate allergic airway sensitization. Changes in $\mathrm{AR}$ and the consequences of RSV infection were correlated with pulmonary inflammation and cytokine production in the peribronchial lymph nodes (the local draining lymph nodes of the lung).

Acute RSV infection resulted in significant increases in AR to $\mathrm{MCh}$, and this was associated with the infiltration of both eosinophils and neutrophils in the lungs. These findings parallel those seen in other models of respiratory viral infection (24). The increases in the response to MCh developed within $4 \mathrm{~d}$ of RSV infection, peaked on day 6 , and resolved by day 21 postinfection. The kinetics of the development of altered AR were paralleled by the cellular infiltration of the lungs with 
eosinophils and neutrophils. The profile of cytokines produced by mononuclear cells from the PBLN during acute infection was mainly of the Th-1 type; IFN- $\gamma$ production was increased, whereas IL-4 and IL-5 production were diminished on day 6 after the infection. By day 12 postinfection, production of these cytokines had returned to normal levels. The increases in IFN- $\gamma$ production during acute RSV infection are in keeping with observations made earlier which showed increased production of total interferon in tracheobroncheal lavages from RSV-infected mice (12). Eosinophil inflammation is usually seen in the context of a Th-2-type cytokine response and not a Th-1-type response as we have observed. In our model it may be brought about by changes in the function of the respiratory epithelium caused by RSV infection such as altered expression of adhesion molecules or an increase in production of chemokines which attract eosinophils. Such changes in epithelial function that affect the recruitment of neutrophils after RSV infection by increasing expression of intercellular adhesion molecule-1 (ICAM-1) (25) and an increase in the production of IL-8 $(26,27)$ have been described in human bronchial epithelial cell lines. An increase in the production of granulocyte macrophage-colony stimulating factor (GM-CSF) (26) in such cell lines after RSV infection could also influence the recruitment of eosinophils. Further, it was reported recently that IFN- $\gamma$ produced by activated $\mathrm{T}$ cells after rhinovirus infection was able to prolong the survival of human peripheral blood eosinophils (28). The increased production of IFN- $\gamma$ after RSV infection may conceivably also prolong the survival of murine pulmonary eosinophils.

As described previously, daily exposure to OVA for $10 \mathrm{~d}$ via the airways results in sensitization of BALB/c mice (21). Whereas this sensitization protocol causes changes in airway function that can be detected by an increased contractile response of tracheal smooth muscle segments to electrical field stimulation, it was not sufficient to trigger MCh-induced changes in AR detectable by barometric whole body plethysmography. Two approaches were used to determine the consequences of RSV infection on subsequent allergic sensitization and AR. First, OVA sensitization was begun on day 11 postinfection when the effects of the acute infection could still be observed. In the second approach, OVA sensitization was begun only after full resolution of the changes induced by acute RSV infection, on day 21 postinfection. In both cases, the effects of airway sensitization were enhanced by a previous RSV infection resulting in increased AR to MCh accompanied by an increase in the accumulation of eosinophils and neutrophils in the lung. Even the brief 5-d exposure to aerosolized OVA, which previously did not prove sufficient to increase reactivity to electrical field stimulation, triggered increased responsiveness to MCh after RSV infection. Inoculation with UV-inactivated RSV did not lead to an enhanced pulmonary inflammation or to increased AR indicating that infectious RSV is required to trigger the changes that persist beyond the effects of acute infection.

How these changes facilitate airway sensitization resulting in the development of airway hyperresponsiveness and enhanced pulmonary inflammation is not clear. Enhancement of airway sensitization to allergen by a preceding respiratory viral infection has been documented in other species but with little characterization of the immune inflammatory changes. Riedel et al. (29) reported enhanced airway responses to specific provocation with OVA in guinea pigs after parainfluenza type
III infection and OVA exposure. A conflicting study in a similar guinea pig model showed impaired airway sensitization after parainfluenza type III infection and the loss of airway hyperresponsiveness (30). In a bovine model, RSV infection enhanced sensitization to an inhaled antigen (31).

Unlike the cytokine response to acute RSV infection, the cytokines produced by mononuclear cells from the PBLN of RSV infected and subsequently OVA-sensitized mice in response to allergen stimulation were mainly of the Th-2 type; the production of IFN- $\gamma$ was reduced whereas IL-4 levels were increased. This suggests that these OVA-specific mononuclear cells of previously RSV-infected mice are more prone to the production of Th- 2 cytokines after allergen exposure. The changes associated with increased AR in these animals could not be explained by increased allergen-specific immunoglobulin production. OVA-specific IgE and IgG1 were detectable in the serum of mice exposed to OVA alone and the levels were not enhanced by RSV infection before sensitization. Our findings differ from other studies that reported increased levels of allergen-specific immunoglobulins after sensitization during respiratory viral infections $(15,16,32,33)$. In these studies, however, animals were exposed to the allergen during the acute infection. Perhaps increased allergen absorption in the lung as a result of inflammation caused by infection resulted in the increases in antibody levels. In our model, mice were exposed to OVA only after the acute phase of the infection and this may account for the lack of differences in OVA-specific immunoglobulin production.

Despite the differences in cytokines produced by cells in the PBLN during acute infection and after OVA sensitization, eosinophils, not neutrophils, appear to play an essential role in the development of altered AR in both situations. This model does not provide evidence directly linking lung eosinophils to airway hyperresponsiveness, but when mice were treated with anti-IL-5 antibody either during the acute infection or concurrent with OVA sensitization, the increases in lung eosinophil numbers were eliminated and AR reverted to normal. There was a significant correlation between eosinophil numbers and Penh values. The increases in lung eosinophils after RSV infection, however, did not correlate with increases in IL-5 production, at least in cultures of PBLN cells. This may indicate sources of IL-5 other than the PBLN cells or that eosinophil influx was induced by a mechanism that we have not defined as yet, such as upregulation of adhesion molecules or altered production of chemokines, such as eotaxin by the respiratory epithelium (25-27). Regardless of the mechanism, anti-IL-5 treatment prevented eosinophil influx into the lungs. Since the numbers of neutrophils were unchanged by this treatment, this implies that the increase in AR in our model is not dependent on neutrophil infiltration. The observed eosinophilia in the lung may well play a critical role in the development of airway hyperresponsiveness, but we cannot exclude that IL-5 had a direct effect on AR that was blocked by the anti-IL-5 treatment. In a model of anti-IL-5 treatment during acute parainfluenza type III infection in the guinea pig, airway hyperresponsiveness was decreased without changes in pulmonary eosinophils, perhaps supporting some direct influence of IL-5 on AR (34). The role of IL-5 and eosinophils in the development of airway hyperresponsiveness in mice is controversial. Foster et al. (35) reported recently that genetically IL-5-deficient B6 mice sensitized by allergen plus adjuvant did not develop bronchial eosinophilia nor increased AR after allergen airway challenge, 
and concluded that IL-5 is essential for both features of allergic airway sensitization in mice. Our own findings (36) studying a model of anti-IL-5 antibody treatment during allergen sensitization via the airways in Balb/c mice support this notion: the anti-IL-5 treatment prevented eosinophil infiltration of the lungs and the development of increased AR. In contrast, Corry et al. (37) reported a lack of effect of anti-IL-5 treatment on increased AR, despite the inhibition of pulmonary eosinophilia in a murine model of airway sensitization, disputing the role of IL-5 in the development of airway hyperresponsiveness. Their findings may be explained by the use of a very low dose ( $8 \mu \mathrm{g}$ in total) of anti-IL-5 antibody and the timing of the treatment, which was given during sensitization only but not during airway challenge.

In summary, we present an animal model that enables the investigation of the effects of RSV infection on subsequent airway sensitization by studying immune mechanisms, airway inflammation, and AR. In this model, RSV induces increases in eosinophil infiltration in the lung that are closely associated with the development of airway hyperresponsiveness both in the acute phase of RSV infection and after subsequent allergic airway sensitization. The pulmonary inflammation after airway sensitization and before RSV infection is associated with a predominant Th-2 cytokine response, in contrast to the predominant Th- 1 cytokine response in the acute phase of RSV infection.

\section{Acknowledgments}

Jürgen Schwarze is a fellow of the Deutsche Forschungsgemeinschaft (Schw 597/1-1).

This work was supported by grant HL-36577 from the National Institutes of Health

\section{References}

1. Empey, D.W., L.A. Laitinen, L. Jacobs, W.M. Gold, and J.A. Nadel. 1976. Mechanisms of bronchial hyperreactivity in normal subjects after upper respiratory tract infection. Am. Rev. Respir. Dis. 113:131-139.

2. Busse, W.W. 1990. Respiratory infections: their role in airway responsiveness and the pathogenesis of asthma. J. Allergy Clin. Immunol. 85:671-683.

3. Cypar, D., J. Stark, and R.F. Lemanske, Jr. 1992. The impact of respiratory infections on asthma. Pediatr. Clin. North Am. 39:1259-1276.

4. McIntosh, K., E.F. Ellis, L.S. Hoffman, T.G. Lybass, J.J. Eller, and V.A. Fulginiti. 1973. The association of viral and bacterial respiratory infections with the exacerbation of wheezing in young asthmatic children. J. Pediatr. 82:578-590.

5. Frick, O.L., D.F. German, and J. Mills. 1979. Development of allergy in children. I. Association with virus infections. J. Allergy Clin. Immunol. 63:228-241.

6. Sigurs, N., R. Bjarnason, F. Sigurbergsson, B. Kjellman, and B. Björkstén. 1995. Asthma and immunoglobulin E antibodies after respiratory syncytial virus bronchiolitis: a prospective cohort study with matched controls. Pediatrics. 95:500-505.

7. Bardin, P.G., S.L. Johnston, and P.K. Pattemore. 1992. Viruses as precipitants of asthma symptoms. II. Physiology and mechanisms. Clin. Exp. Allergy. 22:809-822.

8. Buckner, C.K., V. Songsridej, E.C. Dick, and W.W. Busse. 1985. Studies on the use of the guinea pig as a model for virus provoked airway hyperreactivity. Am. Rev. Respir. Dis. 132:305-310.

9. Folkerts, G., A. Verheyen, and F.P. Nijkamp. 1992. Viral infection in guinea pigs induces a sustained non-specific airway hyperresponsiveness and morphological changes of the respiratory tract. Eur. J. Pharmacol. 228:121-130.

10. Lapin, C.D., P.W. Hiatt, C. Langston, E. Mason, and P.T. Piedra. 1993. A lamb model of human respiratory syncytial virus infection. Pediatr. Pulmonol. 15:151-156.

11. Prince, G.A., A.B. Jenson, R.L. Herswood, E. Camargo, and R.M. Chanock. 1978. The pathogenesis of respiratory syncytial virus infection in cotton rats. Am. J. Pathol. 93:771-783.

12. Anderson, J.J., J. Norden, D. Saunders, G.L. Toms, and R. Scott. 1990. Analysis of the local and systemic immune responses induced in Balb/c mice by experimental respiratory syncytial virus infection. J. Gen. Virol. 71:1561-1570.
13. Graham, B.S., L.A. Bunton, P.F. Wright, and D.T. Karzon. 1991. Role of $\mathrm{T}$ lymphocyte subsets in the pathogenesis of primary infection and rechallenge with respiratory syncytial virus in mice. J. Clin. Invest. 88:1026-1033.

14. Oppenshaw, P.J.M. 1995. Immunity and immunopathology to respiratory syncytial virus. The mouse model. Am. J. Respir. Crit. Care Med. 152:S59-S62.

15. Freihorst, J., P.A. Piedra, J. Okamoto, and P.L. Ogra. 1988. Effect of respiratory syncytial virus infection on the uptake of and immune response to other inhaled antigens. Proc. Soc. Exp. Biol. Med. 188:191-197.

16. Leibovitz, E., J. Freihorst, P.A. Piedra, and P.L. Ogra. 1988. Responses to inhaled ragweed antigen in experimentally induced infection with respiratory syncytial virus. Implication in virally induced allergy. Int. Arch. Allergy Appl. Immunol. 86:112-116.

17. Hamelmann, E., J. Schwarze, K. Takeda, A. Oshiba, G.L. Larsen, C.G. Irvin, and E.W. Gelfand. 1997. Noninvasive measurement of airway responsiveness in allergic mice using barometric plethysmography. Am. J. Resp. Crit. Care Med. In press.

18. Reuman, P.D., S.P. Keely, and G.M. Schiff. 1991. Comparison of class and subclass antibody response to live and UV-inactivated RSV administered intranasally in mice. J. Med. Virol. 35:198-205.

19. Reuman, P.D., S.P. Keely, and G.M. Schiff. 1990. Rapid recovery from RSV infection after combined nasal/oral immunization with killed RSV in mice. J. Med. Virol. 32:67-72.

20. Toms, G.L., P.S. Gardner, C.R. Pullan, M. Scott, and C.E. Taylor. 1980. Secretion of respiratory syncytial virus inhibitors and antibody in human milk throughout lactation. J. Med. Virol. 5:351-360.

21. Larsen, G.L., H. Renz, J.E. Loader, K.L. Bradley, and E.W. Gelfand. 1992. Airway response to electrical field stimulation in sensitized inbred mice. J. Clin. Invest. 89:747-752.

22. Oshiba, A., E. Hamelmann, K. Takeda, K. Bradley, J.E. Loader, G.L. Larsen, and E.W. Gelfand. 1996. Passive transfer of immediate hypersensitivity and airway hyperresponsiveness by allergen-specific immunoglobulin (Ig) E and IgG1 in mice. J. Clin. Invest. 97:1398-1408.

23. Schumacher, J.H., A. O'Garra, B. Shrader, A. van Kimmenade, M.W. Bond, T.R. Mosmann, and R.L. Coffman. 1988. The characterization of four monoclonal antibodies specific for mouse IL-5 and development of mouse and human IL-5 enzyme-linked immunosorbent assay. J. Immunol. 141:1576-1581.

24. Folkerts, G., B. van Esch, M. Janssen, and F.P. Nijkamp. 1992. Virus in duced airway hyperresponsiveness in the guinea pig in vivo: study of bronchoalveolar cell numbers and activity. Eur. J. Pharmacol. 228:219-227.

25. Arnold, R., H. Werchau, and W. König. 1995. Expression of adhesion molecules (ICAM-1, LFA-3) on human epithelial cells (A549) after respiratory syncytial virus infection. Int. Arch. Allergy Immunol. 107:392-393.

26. Noah, T.L., and S. Becker. 1993. Respiratory syncytial virus-induced cytokine production by a human bronchial epithelial cell line. Am. J. Physiol. 265: L472-L478.

27. Arnold, R., B. Humbert, H. Werchau, H. Gallati, and W. König. 1994 Interleukin-8, interleukin-6, and soluble tumour necrosis factor receptor type I release from a human pulmonary epithelial cell line (A549) exposed to respiratory syncytial virus. Immunology. 82:126-133.

28. Gern, J.E., R. Vrtis, E.A.B. Kelly, E.C. Dick, and W.W. Busse. 1996. Rhinovirus produces nonspecific activation of lymphocytes through a monocyte-dependent mechanism. J. Immunol. 157:1605-1612.

29. Riedel, F., A. Krause, W. Slenczka, and C.H.L. Rieger. 1996. Parainfluenza-3-virus infection enhances allergic sensitization in the guinea pig. Clin. Exp. Allergy. 26:603-609.

30. Kudlacz, E.M., and R.W. Knippenberg. 1995. Parainfluenza virus type-3 infection attenuates the respiratory effects of antigen challenge in sensitized guinea pigs. Inflamm. Res. 44:105-110.

31. Gershwin, L.J., S.R. Himes, D.L. Dungworth, S.N. Giri, K.E. Friebertshauser, and M. Camacho. 1994. Effect of bovine respiratory syncytial virus infection on hypersensitivity to inhaled Micropolyspora faeni. Int. Arch. Allergy Immunol. 104:79-91.

32. Sakamoto, M., S. Ida, and T. Taishima. 1984. Effect of influenza virus infection on allergic sensitization to aerosolized ovalbumin in mice. J. Immunol. 132:2614-2617.

33. Holt, P.G., J. Vines, and N. Bilyk. 1988. Effect of influenza virus infection on allergic sensitization to inhaled antigen in mice. Int. Arch. Allergy Appl. Immunol. 86:121-123.

34. Van Osterhout, A.J.M., J. Van Ark, G. Folkerts, H.J. Van der Linde, H.F.J. Savelkoul, A.K.C.P. Verheyen, and F.P. Nijkamp. 1995. Antibody to IL-5 inhibits virus-induced airway hyperresponsiveness to histamine in guinea pigs. Am. J. Respir. Crit. Care Med. 151:177-183.

35. Foster, P.S., S.P. Hogan, A.J. Ramsay, K.I. Matthaei, and I.G. Young. 1996. Interleukin 5 deficiency abolishes eosinophilia, airway hyperreactivity, and lung damage in a mouse model. J. Exp. Med. 183:195-201.

36. Hamelmann, E., A. Oshiba, J. Loader, G.L. Larsen, G. Gleich, J. Lee, and E.W. Gelfand. 1997. Anti-IL-5 antibody prevents airway hyperresponsiveness in a murine model of airway sensitization. Am. J. Respir. Crit. Care Med. 155:819-825.

37. Corry, D.B., H.G. Folkesson, M.L. Warnock, D.E. Erle, M.A. Matthay, J.P. Wiener-Kronish, and R.M. Locksley. 1996. Interleukin 4, but not interleukin 5 or eosinophils, is required in a murine model of acute airway hyperreactivity. J. Exp. Med. 183:109-117. 